\title{
Preliminary Experience of Neuroform Atlas Stenting as a Rescue Treatment after Failure of Mechanical Thrombec- tomy Caused by Residual Intracranial Atherosclerotic Stenosis
}

\author{
Ho Jun $\mathrm{Yi}^{1,2}$ Jae Hoon Sung, ${ }^{1}$ Dong Hoon Lee ${ }^{1}$ \\ Department of Neurosurgery, St. Vincent's Hospital, College of Medicine, The Catholic University of Korea, Suwon, Korea \\ Department of Neurosurgery, ${ }^{2}$ Soonchunhyang University Bucheon Hospital, Bucheon, Korea
}

Objective : The low-profile Neuroform Atlas stent can be deployed directly without an exchange maneuver by navigating into the Gateway balloon. This retrospective study assessed the safety and efficacy of Neuroform Atlas stenting as a rescue treatment after failure of mechanical thrombetomy (MT) for large artery occlusion.

Methods : Between June 2018 and December 2019, a total of 31 patients underwent Neuroform Atlas stenting with prior Gateway balloon angioplasty after failure of conventional MT caused by residual intracranial atherosclerotic stenosis (ICAS). Primary outcomes were successful recanalization and patency of the vessel 24 hours after intervention. Secondary outcomes were vessel patency after 14 days and 3-month modified Rankin Scale. Peri-procedural complications, intracerebral hemorrhage (ICH), and 3-month mortality were reviewed.

Results : With a $100 \%$ of successful recanalization, median value of stenosis was reduced from $79.0 \%$ to $23.5 \%$. Twenty-eight patients (90.3\%) showed tolerable vessel patency after 14 days. New infarctions occurred in three patients (9.7\%) over a period of 14 days; two patient (6.5\%) underwent stent occlusion at 24 hours, and the other patient (3.2\%) with delayed stent occlusion had a non-symptomatic dot infarct. There were no peri-procedural complications. Two patients (6.5\%) developed an ICH immediately after the procedure with one of them is symptomatic.

Conclusion : Neuroform Atlas stenting seems to be an effective and safe rescue treatment modality for failed MT with residual ICAS, by its high successful recanalization rate with tolerable patency, and low peri-procedural complication rate. Further multicenter and randomized controlled trials are needed to confirm our findings.

Key Words : Angioplasty · Atherosclerosis · Thrombectomy · Stents · Stroke $\cdot$ Neuroform atlas.

\section{INTRODUCTION}

Mechanical thrombectomy (MT) is the primary approach used to treat acute ischemic stroke (AIS) caused by large artery occlusion (LAO). MT techniques are constantly evolving, and stent retrievers, contact aspiration, and combined techniques

- Received : May 25, 2020 •Revised : June 23, 2020 •Accepted : July 20, 2020

- Address for reprints : Jae Hoon Sung

Department of Neurosurgery, St. Vincent's Hospital, College of Medicine, The Catholic University of Korea, 93 Jungbu-daero, Paldal-gu, Suwon 16247, Korea Tel : +82-31-249-8985, Fax : +82-31-2649-7020, E-mail : jaehoonsung@gmail.com, ORCID : https://orcid.org/0000-0003-3738-6413 
have resulted in reperfusion rates close to $90 \%{ }^{3,9,11,16)}$. In the era of MT, it is important to determine the appropriate strategy for acute LAO refractory to conventional MT. In such cases, rescue therapies, including balloon angioplasty, stent angioplasty, and intra-arterial glycoprotein IIb/IIIa antagonist injection should be considered ${ }^{13,24,28}$. Moreover, stent angioplasty to restore blood flow should be considered, especially in Asian patients with atherosclerosis-related in situ stenosis or occlusion, because acute LAO with underlying intracranial atherosclerotic stenosis (ICAS) is more common in Asian populations than in Western populations ${ }^{21)}$. Several previous studies have reported outstanding procedural and clinical outcomes for stent angioplasty using self-expanding stents such as the Neuroform (Stryker Neurovascular, Kalamazoo, MI, USA), Enterprise stent (Codman Neurovascular, Raynham, MA, USA), Wingspan (Stryker Neurovascular), or detachable stent retriever Solitaire FR (Covidien, Irvine, CA, USA) in cases with failure of $\mathrm{MT}^{4,7,14,18,21,26)}$.

The Wingspan stent, whose efficacy has been demonstrated in several trials, is the most widely used intracranial stent ${ }^{1,6)}$. It is a self-expanding nitinol stent designed for treatment of severe symptomatic ICAS in coordination with use of the Gateway angioplasty balloon (Boston Scientific, San Leandro, CA, USA $)^{1)}$. Recently, the Wingspan Stent System Post Market Surveillance (WEAVE) trial revealed that use of the Wingspan stent for ICAS was associated with a low peri-procedural complication rate and excellent safety profile ${ }^{1)}$. However, the Wingspan and Gateway balloon system has the significant disadvantage of need to exchange the wire in the device with longer $(300 \mathrm{~cm}, 0.014 \mathrm{inch})$ wire. In addition, one of the reasons for poor outcomes in the Stenting and Aggressive Medical Management for Preventing Recurrent stroke in Intracranial Stenosis (SAMMPRIS) trial was occurrence of ipsilateral cerebral hemorrhage, which may be associated with the intracranial exchange maneuver ${ }^{6}$. The newly developed, low-profile Neuroform Atlas (Stryker Neurovascular, Fremont, CA, USA) is a nitinol self-expanding, hybrid/open cell stent that can be delivered through a 0.017 -inch compatible microcathe$\operatorname{ter}^{5}$. This stent can be deployed directly by navigating into the Gateway balloon without an exchange maneuver and offers improved navigability and better conformability and stability, especially in tortuous vascular anatomy ${ }^{19)}$. For these reasons, the authors performed permanent stenting with a Neuroform Atlas stent and prior balloon angioplasty in cases with refrac- tory to conventional MT due to residual ICAS. The purpose of this retrospective study was to evaluate the safety and efficacy of this new device as a rescue treatment modality after failure of MT for LAO with underlying ICAS.

\section{MATERIALS AND METHODS}

\section{Study population and imaging follow up}

This study, based on prospectively collected data and retrospective review, was approved by the Institutional Review Board of St. Vincent's Hospital (IRB No. VC20RISI0067) of each participating center. Between June 2018 and December 2019, 214 cases of MT for LAO were performed, and the Neuroform Atlas stent was used to treat 31 cases of failed conventional MT with residual ICAS. All of intracranial stenting in this study was performed with the Neuroform Atlas stent and prior balloon angioplasty with the Gateway balloon, and the neurointerventionalists decided to use the Neuroform Atlas stent based on clinical symptoms and angiographic findings of each patient. Target arteries for stenting were as follows : distal internal carotid artery (ICA), middle cerebral artery (MCA) M1 segment, and basilar artery (BA). Percentage stenosis of the target lesion was measured as the most severe stenosis with reference to the normal luminal diameter of the target arterial segment by digital subtraction angiography. All procedures were performed under conscious sedation with continuous blood pressure (BP) monitoring to avoid intra-procedural hypotension or hypertension. During the post-procedural period, patients were typically monitored in Neuro Intensive Care units for at least 24 hours for critical care, with BP monitoring to reduce the risk for reperfusion hemorrhage. All patients underwent computed tomography (CT) immediately after the intervention to evaluate hemorrhage, and vessel status with occurrence of hemorrhage was evaluated by computed tomography angiography with perfusion (CTAP) map at 24 hours after the procedure (CTAP imaging protocol is described in Supplementary Fig. $1)^{23}$. Fourteen days after the procedure, magnetic resonance angiography (MRA) with diffusion-weighted imaging (DWI) was performed to evaluate vessel status and occurrence of new ischemic stroke.

\section{Neuroform Atlas stent system}

Neuroform Atlas is a self-expanding nitinol stent with a 
mixed open-cell/closed-cell design. The stent can be delivered via a 0.0165 inch or 0.017 inch microcatheter. Images of the Neuroform Atlas stent are provided in Fig. 1; note the three radiopaque markers at each end. The stent has an open-cell structure through most of its length, with the exception of its

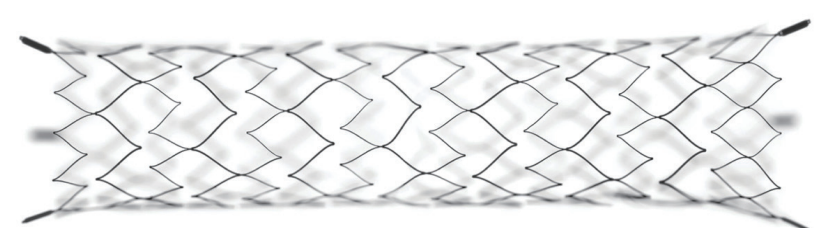

Fig. 1. Illustration of the Neuroform Atlas stent with W-shaped cells and alternating cell count per row. Pictures were provided courtesy of Stryker. closed-cell proximal end, which increases device stability when re-crossing with a microcatheter ${ }^{19}$. The mix of open and closed cells in the stent design is supposed to improve stability within the vessel via the closed cells at the proximal end while providing high flexibility, even in tortuous vessels, through the open cells in the middle. Owing to the mostly open-cell design, re-sheathing of the stent is not possible ${ }^{12)}$. There is very little or no foreshortening with stent release, which improves the accuracy of stent placement. Available stent lengths are 15, 21, 24, and $30 \mathrm{~mm}$, and stent diameter ranges from $3 \mathrm{~mm}$ to $4.5 \mathrm{~mm}$; these stents can be placed in vessels with diameters ranging from 2 to $4.5 \mathrm{~mm}^{20)}$.
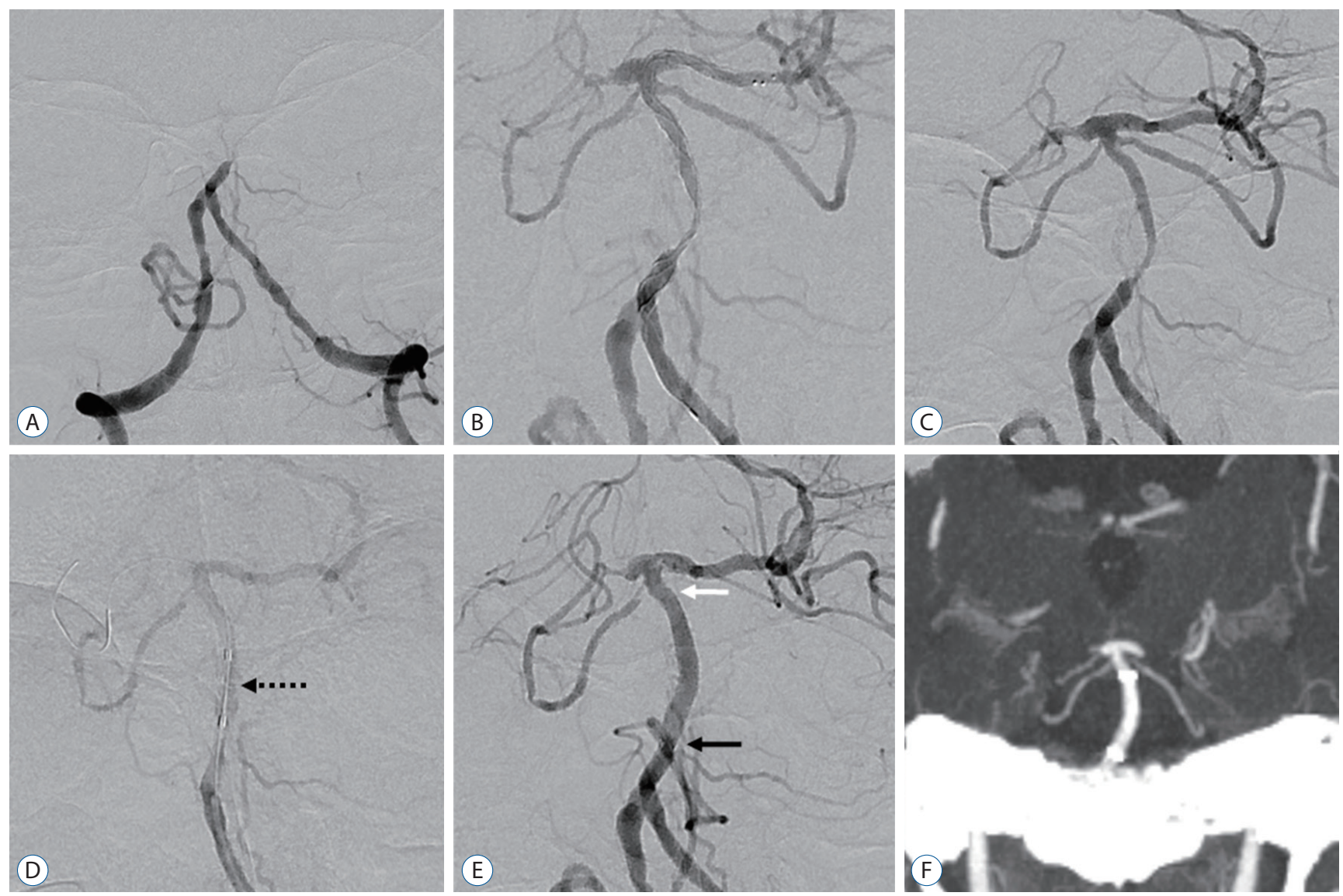

Fig. 2. An example of a case with balloon angioplasty and intracranial stenting using the Neuroform Atlas stent followed by mechanical thrombectomy for basilar artery (BA) occlusion with stenosis. A : Initial angiography revealed BA trunk occlusion. B : Severe stenosis of the BA trunk was confirmed by angiography with the Trevo stent retriever deployed. C : Angiography after Trevo stent retrieval also showed BA trunk stenosis status. D : Balloon angioplasty was performed for the severe stenotic lesion of BA trunk with a 2.5×15 mm sized Gateway balloon (black dot arrow : Gateway balloon). E : Serially, a $3.0 \times 21 \mathrm{~mm}$-sized Neuroform Atlas stent was delivered directly through the lumen of the angioplasty Gateway balloon. Follow-up angiography after Neuroform Atlas stent deployment revealed significantly improved BA trunk stenosis (white and black arrows : distal and proximal markers of the Neuroform Atlas stent, respectively). F : Maximum intensity projection coronal image of computed tomography angiography with perfusion performed 24 hours after the intervention showed tolerable BA flow with the Neuroform Atlas stent. 


\section{Endovascular technique}

After failure of conventional MT for LVO with AIS, intracranial Neuroform Atlas stenting and prior Gateway balloon angioplasty was performed as a rescue therapy in cases with identified residual stenosis greater than 70\% during MT. Inclusion criteria as follows : 1) identified residual ICAS greater than $70 \%$ with re-occlusion after MT and 2) insufficient distal flow restoration despite recanalization after MT. Prior to thrombectomy, intravenous (IV) tissue plasminogen activator (t-PA, alteplase) was administered within 4.5 hours after stroke onset at a maximum dose of $0.9 \mathrm{mg} / \mathrm{kg}$ in accordance with the European Cooperative Acute Stroke Study (ECASS) III trial ${ }^{10)}$. MT with Solitaire Platinum (Medtronic, Dublin, Ireland) or Trevo XP Provue (Stryker Neurovascular, Fremont) stent retriever was performed to the LAO. In 20 patients (64.5\%), simultaneous aspiration was applied for combined technique with 5Fr SOFIA (Sofia 5, MicroVention-Terumo, Tustin, CA, USA) or 6Fr AXS Catalyst 6 (CAT6, Stryker Neurovascular, Mountain View, CA, USA). An 8Fr balloon-guiding catheter (BGC) (FlowGate2, FG2; Stryker Neurovascular,
Fremont) was used in 24 patients (77.4\%). All patients received heparin with initiation of intervention, and 22 patients $(70.9 \%)$ received intra-arterial (IA) tirofiban over 5 minutes $(0.05 \mathrm{mg} / \mathrm{min})$ before stenting, for residual thrombus after MT. In contrast, a loading dose of dual antiplatelet medications (300 mg aspirin and $300 \mathrm{mg}$ clopidogrel) were applied in patients who have not used IA tirofiban, immediately before the balloon angioplasty and intracranial stenting. Under roadmap guidance, an overthe-wire balloon (Gateway balloon) was to pass the lesion with 0.014 microwire $(200 \mathrm{~cm})$. Balloon angioplasty was performed with the nominal diameter at 6 atmospheres set to $80 \%$ of the true luminal diameter or about $60 \%$ of the luminal diameter in lesions directly adjacent to visible perforators ${ }^{8)}$. Under-dilation of balloon is recommended to avoid arterial dissection, vessel rupture, and the snowplow effect of compressed plaque on perforator arteries ${ }^{1)}$. After performing angioplasty of the stenosis with the Gateway balloon, the Neuroform Atlas stent was delivered directly through the lumen of the Gateway balloon without a need for an intracranial exchange maneuver. Stent diameter was at least $1 \mathrm{~mm}$ wider than that of the target
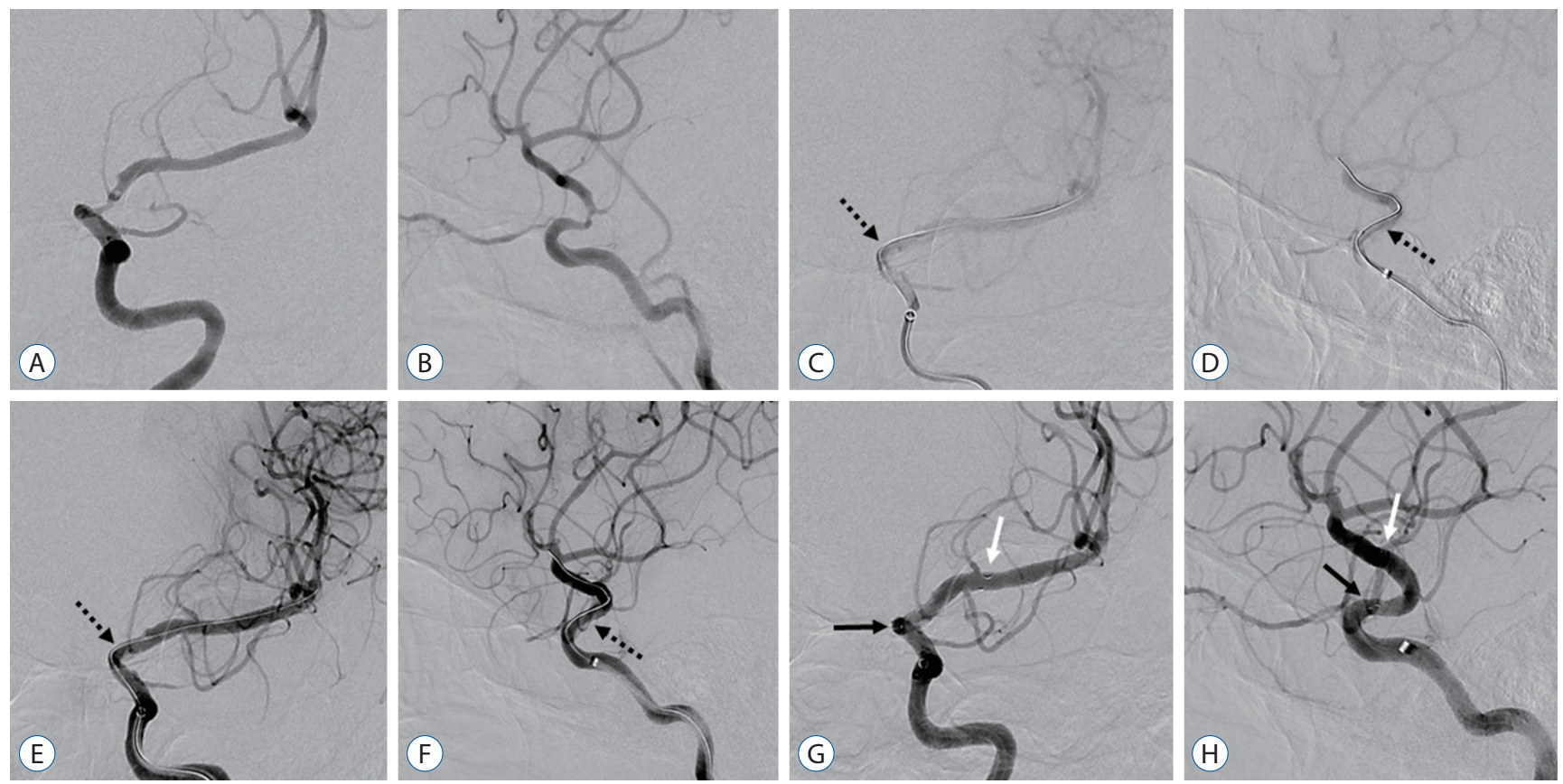

Fig. 3. Representative case of balloon angioplasty with the Gateway balloon and Neuroform Atlas stent deployment followed by mechanical thrombectomy in internal carotid artery (ICA) stenosis with occlusion. A and B : After mechanical thrombetomy with the stent retriever, follow-up angiography showed severe stenosis of the left distal ICA. C and D : Over-the-wire balloon (3.0×15 mm sized Gateway balloon) was delivered to the lesion of left distal ICA stenosis by Traxcess micro-wire guidance (black dotted arrow : Gateway balloon). E and F : Balloon angioplasty at the stenotic lesion was performed with a Gateway balloon (black dotted arrow : Gateway balloon). G and H : Neuroform Atlas stent (4.0×24 mm) was delivered directly through the lumen of the angioplasty balloon without the need for an intracranial exchange maneuver (white and black arrows : distal and proximal markers of the Neuroform Atlas stent, respectively). 
vessel, and the exceeding length of the stent was at least $5 \mathrm{~mm}$ longer than that of the target lesion (see illustrative cases in Figs. 2 and 3). During the post-thrombectomy period, if there was no evidence of hemorrhage on the CT scan performed immediately after procedure, a maintenance dose of $0.05 \mu \mathrm{g} /$ $\mathrm{kg} / \mathrm{min}$ of tirofiban was pumped intravenously during 24 hours, in patients who used IA tirofiban. Patients received dual antiplatelet therapy with daily aspirin $(100 \mathrm{mg})$ and clopidogrel $(75 \mathrm{mg})$, in addition to a statin during the post procedural period. Seven days after intervention, reactivity toward antiplatelet therapy was assessed with the P2Y12 test (VerifyNow; Accumetrics, San Diego, CA, USA) to identify optimal therapeutic platelet reactivity $(95 \leq$ platelet reactivity unit [PRU] $\leq 219$ ), and medication changes were permitted for patients with low platelet reactivity (PRU <95) or high platelet reactivity $(\text { PRU }>219)^{22)}$.

\section{Outcome and complications}

Multivariable factors of patient sex, age, risk factors (hypertension [HTN], diabetes mellitus [DM], atrial fibrillation [AF], coronay artery disease $[\mathrm{CAD}]$, smoking), prior stroke or transient ischemic attack (TIA), lipid profiles (high-density lipoprotein [HDL] cholesterol, low-density lipoprotein [LDL] cholesterol, triglyceride [TG]), application of IV t-PA, value of PRU, application of IA or IV tirofiban, site of arterial occlusion or stenosis, National Institutes of Health Stroke Scale (NIHSS) score (range, 0-42 with a higher score indicating more severe neurologic deficit), and modified Rankin Scale (mRS) score at 3 months were reviewed. Procedure time was defined as the time from groin puncture to MT and balloon angioplasty with stenting. Procedural details were evaluated according to device and comprised rate of pre- and post-stenting stenosis and pressure and duration of Gateway balloon angioplasty. Primary outcomes were evaluated as successful recanalization, which was defined as thrombolysis in cerebral infarction (TICI) scores of $2 \mathrm{~b}$ or $3^{27}$, and patency of the intracranial vessel after stenting, which was evaluated by CTAP 24 hours after intervention. Secondary outcome of intracranial vessel patency as revealed by MRA 14 days from the intervention and the 3-month mRS was also evaluated. Various complications, such as peri-procedural complication (in-stent occlusion or thrombus, arterial dissection or perforation), intracerebral hemorrhage (ICH), new infarction within 14 days, and 3-month mortality were reviewed. All multimodal factors and clinical data were analyzed by all authors.

\section{Statistical analyses}

All data were analyzed using Stata Statistical software, version 15 (Stata, College Station, TX, USA). Categorical variables are expressed as absolute values (percentages), and quantitative variables as mean ( \pm standard deviation $[\mathrm{SD}]$ ) or median (range), as appropriate.

Table 1. Demographic factors

\begin{tabular}{|c|c|}
\hline Baseline characteristic & Total $(n=31)$ \\
\hline Female & $11(35.5)$ \\
\hline Age (years) & $66.4 \pm 12.9$ \\
\hline Hypertension & $18(58.1)$ \\
\hline Diabetes mellitus & $11(35.5)$ \\
\hline Atrial fibrillation & $7(22.6)$ \\
\hline Coronary artery disease & $5(16.1)$ \\
\hline Smoking & $8(25.8)$ \\
\hline $\begin{array}{l}\text { Prior stroke or transient ischemic } \\
\text { attack }\end{array}$ & 7 (22.6) \\
\hline Dyslipidemia & $16(51.6)$ \\
\hline Cholesterol (mmol/L) & $169.2 \pm 43.2$ \\
\hline $\mathrm{HDL}(\mathrm{mmol} / \mathrm{L})$ & $49.1 \pm 11.8$ \\
\hline LDL (mmol/L) & $102.9 \pm 31.1$ \\
\hline Triglyceride (mmol/L) & $94.3 \pm 41.5$ \\
\hline Body mass index & $29.6 \pm 5.6$ \\
\hline Left hemisphere stroke & $14(45.2)$ \\
\hline Tissue-plasminogen activator & $9(29.0)$ \\
\hline Initial NIHSS & $5.6 \pm 3.4,4.5(1,14)$ \\
\hline Baseline $\mathrm{mRS} *$ & $0.6(0.6), 0.0(0,2)$ \\
\hline \multicolumn{2}{|l|}{ Target artery } \\
\hline Middle cerebral artery & $18(58.1)$ \\
\hline Distal internal carotid artery & $5(16.1)$ \\
\hline Basilar artery & $8(25.8)$ \\
\hline \multicolumn{2}{|l|}{ Anti-thrombotics } \\
\hline Intra-arterial tirofiban & $22(70.9)$ \\
\hline Intravenous tirofiban & $21(67.7)$ \\
\hline PRU & $205.7 \pm 50.9,205.0(98,286)$ \\
\hline
\end{tabular}

Values are presented as mean \pm standard deviation, median (range [min, max]), or number (\%). *Ranges from 0 to 6. HDL : high-density lipoprotein cholesterol, LDL : low-density lipoprotein cholesterol, NIHSS : National Institutes of Health Stroke Scale, mRS : modified Rankin Scale, $\mathrm{PRU}$ : platelet reactivity unit (P2Y12 result) 


\section{RESULTS}

\section{Demographic data}

Eleven patients (35.5\%) were female, and the mean age of patients was 66.4 years (SD, 12.9). The rates of HTN, DM, AF, CAD, smoking, prior stroke or TIA, and dyslipidemia were $58.1 \%, 35.5 \%, 22.6 \%, 16.1 \%, 25.8 \%, 22.6 \%$, and $50.0 \%$, respectively. Mean lipid levels were as follows : cholesterol 169.2 mmol/L (SD, 43.2), HDL 49.1 mmol/L (SD, 11.8), LDL 102.9 $\mathrm{mmol} / \mathrm{L}$ (SD, 31.1), and TG $94.3 \mathrm{mmol} / \mathrm{L}$ (SD, 41.5). Mean body mass index was 29.6 (SD, 5.6), and 14 patients (42.2\%) experienced left hemisphere stroke. In nine patients $(29.0 \%)$, IV t-PA was applied prior to intervention. Mean initial NIHSS was $5.6(\mathrm{SD}, 3.4$; median, 4.5; range, 1-14), and baseline mRS

Table 2. Procedural details and outcomes

\begin{tabular}{lc}
\hline Procedural detail & Total $(\mathbf{n = 3 1 )}$ \\
\hline Interventionalists (A : B) & $18: 13$ \\
\hline Use of BGC & $24(77.4)$ \\
\hline Combined technique & $20(64.5)$ \\
Stent retriever (Solitaire : Trevo) & $15: 16$ \\
\hline Procedure time (minutes) & $59.0 \pm 17.5,52(39,152)$ \\
Baseline stenosis (\%) & $80.2 \pm 4.4,79.0(74,88)$ \\
Post stenting stenosis (\%) & $23.8 \pm 6.8,23.5(13,41)$ \\
Balloon inflation pressure (atm) & $6.4 \pm 2.6,6.0(4,12)$ \\
Balloon inflation duration (seconds) & $64.7 \pm 32.4,55.0(15,180)$ \\
Outcomes & \\
Successful recanalization* & $31(100.0)$ \\
CTAP follow up & $31(100.0)$ \\
CTAP patency at 24 hours & $29(93.5)$ \\
MRA patency at 14 days & $28(90.3)$ \\
3 months mRS & \\
Complications & $1.6 \pm 1.7,1.0(0,6)$ \\
Peri-procedural & \\
Stent occlusion or in-stent thrombus & $0(0.0)$ \\
Arterial dissection & $0(0.0)$ \\
Vessel perforation & $0(0.0)$ \\
Intracerebral hemorrhage & $0(0.0)$ \\
Symptomatic intracerebral hemorrhage & $2(6.5)$ \\
14 days new infarction & $1(3.2)$ \\
3 months mortality & $3(9.7)$ \\
\hline
\end{tabular}

Values are presented as mean \pm standard deviation, median (range [min, max]), or number (\%). *Thrombolysis in cerebral infarction 2b or 3. ${ }^{\dagger}$ Ranges from 0 to 6. BGC: balloon guide catheter, CTAP : computed tomography angiography with perfusion, MRA : magnetic resonance angiography, mRS : modified Rankin Scale was 0.6 (SD, 0.6; median, 0 ; range, $0-2$ ). Target artery distribution was the MCA in 18 patients (58.1\%), distal ICA in five patients (16.1\%), and BA in eight patients (25.8\%). The application rates of IA and IV tirofiban were $70.9 \%$ (22 patients) and $67.7 \%$ (21 patients), respectively. Mean PRU value was 205.7 (SD, 50.9; median, 205.0; range, 98-286) (Table 1).

\section{Procedural details, outcomes and complications}

One interventionalist (A) performed 18 procedures, while the others were performed by a second interventionalist (B). BGC was used in 24 patients (77.4\%), and 20 patients (64.5\%) underwent MT with the combined technique. Fifteen Solitaire and 16 Trevo stent receivers were used. Median procedure time was 52 minutes (range, 39-152). Mean baseline stenosis of the target artery was $80.2 \%$ (SD, 4.4; median, 79.0; range, 74-88), and mean post-stenting stenosis was $23.8 \%$ (SD, 6.8; median, 23.5; range, 13-41). Mean balloon inflation pressure was $6.4 \mathrm{~atm}(\mathrm{SD}, 2.6)$, and median balloon inflation pressure was $6.0 \mathrm{~atm}$, with a range of $4-12 \mathrm{~atm}$. The mean and median values of balloon inflation time were 64.7 seconds (SD, 32.4) and 55.0 seconds (range, 15-150), respectively. Successful recanalization was achieved in all patients (100\%), and CTAP follow-up was performed 24 hours after the procedure. Twenty-nine of the 31 patients (93.5\%) showed tolerable vessel patency with perfusion status 24 hours after stenting. MRA was performed after 14 days, and vessel patency of the peri-stent was good at 28 patients (90.3\%). Overall, stent occlusion was observed in three patients during 14 days. There were no periprocedural complications, such as stent occlusion, in-stent thrombus, dissection, or vessel perforation. In two patients (6.5\%), an ICH developed immediately after the procedure, but only one case $(3.2 \%)$ is symptomatic. Three patients (9.7\%) had a new infarction on DWI at 14 days after the procedure : two of these patients underwent stent occlusion on CTAP at 24 hours, while the other patient had a non-symptomatic dot infarct even though stent occlusion was showed on the MRA at 14 days. One patient died of ICH, and other died of a medical condition within 3 months after the intervention ( $6.5 \%$ of 3-month mortality rate) (Table 2).

\section{DISCUSSION}

The results of this study indicate that, in patients with fail- 
ure of MT caused by residual ICAS, permanent stenting with a Neuroform Atlas stent with prior Gateway balloon angioplasty resulted in $100 \%$ successful recanalization. There were no peri-procedural complications, and only three patients (9.7\%) underwent stent occlusion, while the other 28 (90.3\%) showed tolerable vessel patency during 14 days after the procedure. In addition, there was only one case of symptomatic ICH (3.2\%). These findings suggest that Neuroform Atlas stenting followed by Gateway balloon angioplasty is a safe and effective procedure and could be a rescue treatment modality for ICAS refractory to conventional MT.

The efficacy and safety of permanent stenting with various stents, Solitaire FR or self-expandable stents, such as the Enterprise, Neuroform, and Wingspan for recanalization of acute stroke have been evaluated in several previous studies $^{2,4,15,17,21)}$. In these studies, successful recanalization rates ranged from $83.3 \%$ to $100 \%$, and $\mathrm{ICH}$ occurred in 5.6 to $11.3 \%$ of cases. Although different classification criteria were applied to each study, the results of our study (successful recanalization rate of $100 \%$, symptomatic ICH rate of $3.2 \%$ ) are not inferior to those reported in previous studies. Compared to other studies for stent angioplasty in symptomatic ICAS without acute occlusion, the SAMMPRIS trial with Wingspan stent revealed a high rate of perioperative complications such as peri-procedural stroke, bleeds, and death rate of $14.7 \%{ }^{6}$. The recently announced WEAVE trial with Wingspan stent reported an event-free rate of $97.4 \%$ over 72 hours with a nonfatal stroke rate of $1.3 \%$ and mortality rate of $1.3 \%{ }^{1)}$. Median stenosis value in our study and the WEAVE trial decreased from $79.0 \%$ to $23.5 \%$ and from $82.0 \%$ to $27.5 \%$, respectively. It is difficult to directly compare the results of the SAMMPRIS and WEAVE trials that involved antiplatelet pre-medication and well-planned treatment with those of our study where we performed emergency intervention for refractory occlusion or residual ICAS after conventional MT. However, our study showed non-inferior results compared to the SAMMPRIS and WEAVE trials with regard to peri-procedural complications and other results, even though we performed our procedures without antiplatelet pre-medication.

The main advantage of permanent stenting with the Neuroform Atlas is that it can be deployed directly into the Gateway balloon without an exchange maneuver. This simple procedure is possible because the Neuroform Atlas stent can be delivered via a 0.0165 inch or 0.017 inch microcatheter and has high flexibility and navigability ${ }^{5,19,20)}$. In addition, accurate stent deployment is possible with little stent shortening and expansion failure due to the open-cell structure of the stent through most of its length, with the exception of the closedcell proximal end ${ }^{20)}$. Wingspan stent, which is widely used in ICAS treatment, has a high radial force and is more rigid than the Neuroform Atlas stent ${ }^{29)}$. Wingspan stent also requires exchange maneuvers through long wires after Gateway balloon angioplasty. These characteristics can create various procedure-related complications during Wingspan stenting, and some studies have shown that the learning curve is a factor influencing successful Wingspan stenting ${ }^{1,25)}$. Complex ICAS cases, such as those with tortuous vascular segments, long (>15 mm) lesions, and arterial bifurcations are associated with higher rates of complications and render Wingspan stenting particularly difficult ${ }^{7)}$. Use of Neuroform Atlas stenting in ICAS can reduce these peri-procedural complications even for interventionalists who do not have extensive experience by allowing smoother navigation with no exchange maneuver.

There are a few technical considerations and need for cautions in Neuroform Atlas stenting through Gateway balloon. First, the stability of device may be deteriorated in tortuous or angulated vessel, because recent version of Neuroform Atlas stent has no tip. In these cases, better stability can be achieved by using an intermediate catheter. Second, re-sheating or adjustment of stent position after deployment was impossible due to open-cell characteristic of stent. Furthermore, re-passage of gateway balloon through deployed Neuroform Atlas stent for post-stenting balloon angioplasty is not possible caused by its open cell without tip nature. Third, distal marker of Gateway balloon is not the real distal end of balloon catheter but the distal part of balloon, so the proximal marker of Neuroform Atlas stent should be passed far more than distal marker of gateway balloon, to be fully deployed.

The major limitations of this study are its relatively small sample size and its retrospective, non-blinded nature. We had described an off-label application of Neuroform Atlas stent for intracranial stenting as a rescue therapy in failure of MT with identified residual stenosis, and Gateway balloon is not designed to deploy a Neuroform Atlas stent. However, this innovative off-label technique revealed its effectiveness and safety in our study. Heparinization was performed immediately before the procedure, but we did not analyze activated clotting time. Longer-term angiographic follow-up data for restenosis, 
in-stent thrombosis, and stent occlusion were not obtained. Furthermore, other potential unmeasured confounding variables were not controlled for, although every effort was made to adjust for the possibility of spurious results. Nevertheless, we described a procedure to treat ICAS with the Neuroform Atlas stent through Gateway angioplasty balloon without an exchange maneuver. In addition, we presented a method to treat ICAS accompanied by acute occlusion with a focus on peri-procedural safety and patency of blood flow during the acute period. We are currently conducting additional longterm clinical and radiological follow-up studies.

\section{CONCLUSION}

In cases with failure of MT caused by residual ICAS, Neuroform Atlas stenting could be an effective rescue modality for maintaining distal flow, with its high successful recanalization rate and tolerable patency. Furthermore, it had a low peri-procedural complication rate, even in emergency procedures without pre-antiplatelet medication. Our results indicate that the Neuroform Atlas stenting is another treatment option for patients who have residual ICAS with acute occlusion. The unique characteristics of the Neuroform Atlas stent enable its direct delivery through the lumen of the Gateway angioplasty balloon, without the need for an intracranial exchange maneuver. Future long-term follow-up results and prospective multicenter, randomized controlled trials are needed to corroborate our findings.

\section{CONFLICTS OF INTEREST}

No potential conflict of interest relevant to this article was reported.

\section{INFORMED CONSENT}

This type of study does not require informed consent.

\section{AUTHOR CONTRIBUTIONS}

\author{
Conceptualization : HJY, JHS \\ Data curation : HJY \\ Formal analysis : $\mathrm{HJY}$ \\ Methodology : HJY \\ Project administration : HJY, JHS \\ Visualization : HJY \\ Writing - original draft : HJY \\ Writing - review \& editing : HJY, JHS, DHL
}

\section{ORCID}
Ho Jun Yi
https://orcid.org/0000-0003-3061-0689
Jae Hoon Sung
https://orcid.org/0000-0003-3738-6413
Dong Hoon Lee
https://orcid.org/0000-0002-3796-8707

\section{- Supplementary materials}

The online-only data supplement is available with this article at https://doi.org/10.3340/jkns.2020.0146.

\section{References}

1. Alexander MJ, Zauner A, Chaloupka JC, Baxter B, Callison RC, Gupta R, et al. : WEAVE trial: final results in 152 on-label patients. Stroke 50 : 889-894, 2019

2. Baek JH, Kim BM, Kim DJ, Heo JH, Nam HS, Yoo J : Stenting as a rescue treatment after failure of mechanical thrombectomy for anterior circulation large artery occlusion. Stroke $47:$ 2360-2363, 2016

3. Bracard S, Ducrocq X, Mas JL, Soudant M, Oppenheim C, Moulin T, et al. : Mechanical thrombectomy after intravenous alteplase versus alteplase alone after stroke (THRACE): a randomised controlled trial. Lancet Neurol 15 : 1138-1147, 2016

4. Brekenfeld C, Schroth G, Mattle HP, Do DD, Remonda L, Mordasini P, et al. : Stent placement in acute cerebral artery occlusion: use of a selfexpandable intracranial stent for acute stroke treatment. Stroke 40 : 847-852, 2009

5. Ciccio G, Robert T, Smajda S, Fahed R, Desilles JP, Redjem H, et al. : Double stent assisted coiling of intracranial bifurcation aneurysms in $Y$ and $X$ configurations with the Neuroform Atlas stent: immediate and mid term angiographic and clinical follow-up. J Neurointerv Surg 11 : 1239-1242, 2019

6. Derdeyn $\mathrm{CP}$, Chimowitz MI, Lynn MJ, Fiorella D, Turan TN, Janis LS, et al. : Aggressive medical treatment with or without stenting in high-risk 
patients with intracranial artery stenosis (SAMMPRIS): the final results of a randomised trial. Lancet 383 : 333-341, 2014

7. Feng Z, Duan G, Zhang P, Chen L, Xu Y, Hong B, et al. : Enterprise stent for the treatment of symptomatic intracranial atherosclerotic stenosis: an initial experience of 44 patients. BMC Neurol $15: 187,2015$

8. Fiorella D, Levy El, Turk AS, Albuquerque FC, Niemann DB, AagaardKienitz $B$, et al. : US multicenter experience with the wingspan stent system for the treatment of intracranial atheromatous disease: periprocedural results. Stroke $38: 881-887,2007$

9. Goyal M, Menon BK, van Zwam WH, Dippel DW, Mitchell PJ, Demchuk AM, et al. : Endovascular thrombectomy after large-vessel ischaemic stroke: a meta-analysis of individual patient data from five randomised trials. Lancet 387 : 1723-1731, 2016

10. Hacke W, Kaste M, Bluhmki E, Brozman M, Dávalos A, Guidetti D, et al. : Thrombolysis with alteplase 3 to 4.5 hours after acute ischemic stroke. N Engl J Med 359 : 1317-1329, 2008

11. Humphries W, Hoit D, Doss VT, Elijovich L, Frei D, Loy D, et al. : Distal aspiration with retrievable stent assisted thrombectomy for the treatment of acute ischemic stroke. J Neurointerv Surg 7 : 90-94, 2015

12. Jankowitz BT, Hanel R, Jadhav AP, Loy DN, Frei D, Siddiqui AH, et al. : Neuroform Atlas Stent System for the treatment of intracranial aneurysm: primary results of the Atlas Humanitarian Device Exemption cohort. J Neurointerv Surg $11: 801-806,2019$

13. Leischner H, Flottmann F, Hanning U, Broocks G, Faizy TD, Deb-Chatterji $M$, et al. : Reasons for failed endovascular recanalization attempts in stroke patients. J Neurointerv Surg 11 : 439-442, 2019

14. Levy El, Rahman M, Khalessi AA, Beyer PT, Natarajan SK, Hartney ML, et al. : Midterm clinical and angiographic follow-up for the first Food and Drug Administration-approved prospective, single-arm trial of primary stenting for stroke: SARIS (stent-assisted recanalization for acute ischemic stroke). Neurosurgery 69 : 915-920; discussion 920, 2011

15. Levy El, Siddiqui AH, Crumlish A, Snyder KV, Hauck EF, Fiorella DJ, et al. : First Food and Drug Administration-approved prospective trial of primary intracranial stenting for acute stroke: SARIS (stent-assisted recanalization in acute ischemic stroke). Stroke 40 : 3552-3556, 2009

16. Maegerlein C, Mönch S, Boeckh-Behrens $T$, Lehm M, Hedderich DM, Berndt MT, et al. : PROTECT: PRoximal balloon Occlusion TogEther with direCt Thrombus aspiration during stent retriever thrombectomy - evaluation of a double embolic protection approach in endovascular stroke treatment. J Neurointerv Surg $10: 751-755,2018$

17. Mocco J, Hanel RA, Sharma J, Hauck EF, Snyder KV, Natarajan SK, et al. : Use of a vascular reconstruction device to salvage acute ischemic occlu- sions refractory to traditional endovascular recanalization methods. J Neurosurg 112 : 557-562, 2010

18. Salik AE, Selcuk HH, Zalov H, Kilinc F, Cirak M, Kara B : Medium-term results of undersized angioplasty and stenting for symptomatic high-grade intracranial atherosclerotic stenosis with enterprise. Interv Neuroradiol 25 : 484-490, 2019

19. Tsai JP, Hardman J, Moore NZ, Hussain MS, Bain MD, Rasmussen PA, et al. : Early post-humanitarian device exemption experience with the Neuroform Atlas Stent. J Neurointerv Surg 11 : 1141-1144, 2019

20. Ulfert C, Pham M, Sonnberger M, Amaya F, Trenkler J, Bendszus M, et al. : The Neuroform Atlas Stent to assist coil embolization of intracranial aneurysms: a multicentre experience. J Neurointerv Surg 10 : 11921196, 2018

21. Woo HG, Sunwoo L, Jung C, Kim BJ, Han MK, Bae HJ, et al. : Feasibility of permanent stenting with solitaire $F R$ as a rescue treatment for the reperfusion of acute intracranial artery occlusion. AJNR Am J Neuroradiol 39 : 331-336, 2018

22. Yi HJ, Hwang G, Lee $B H$ : Variability of platelet reactivity on antiplatelet therapy in neurointervention procedure. J Korean Neurosurg Soc 62 : 3-9, 2019

23. Yi HJ, Sung JH, Lee DH, Yang SH, Hong JT : A useful diagnostic method to reduce the in-hospital time delay for mechanical thrombectomy: volume perfusion computed tomography with added vessel reconstruction. J Neurosurg, 2018 [Epub ahead of print]

24. Yoon W, Kim SK, Park MS, Kim BC, Kang HK : Endovascular treatment and the outcomes of atherosclerotic intracranial stenosis in patients with hyperacute stroke. Neurosurgery 76 : 680-686; discussion 686, 2015

25. Yu SC, Leung TW, Lee KT, Wong LK : Learning curve of Wingspan stenting for intracranial atherosclerosis: single-center experience of 95 consecutive patients. J Neurointerv Surg 6 : 212-218, 2014

26. Zaidat 00, Wolfe T, Hussain SI, Lynch JR, Gupta R, Delap J, et al. : Interventional acute ischemic stroke therapy with intracranial self-expanding stent. Stroke 39 : 2392-2395, 2008

27. Zaidat 00, Yoo AJ, Khatri P, Tomsick TA, von Kummer R, Saver JL, et al. : Recommendations on angiographic revascularization grading standards for acute ischemic stroke: a consensus statement. Stroke 44 : 2650 2663, 2013

28. Zhao H, Zhang J, Gu D, Shi Z, Pan J, Geng Y, et al. : Tirofiban facilitates the reperfusion process during endovascular thrombectomy in ICAS. Exp Ther Med 14 : 3314-3318, 2017

29. Zhao LB, Park $S$, Lee $D$, Lee $D H$, Suh DC : Mechanism of procedural failure related to wingspan. Neurointervention $7: 102-108,2012$ 